\title{
Effect of some solid properties on gas-liquid mass transfer in a bubble column
}

\author{
P. Mena ${ }^{a}$, A. Ferreira ${ }^{a, b}$, J.A. Teixeira ${ }^{b}$, F. Rocha ${ }^{a, *}$

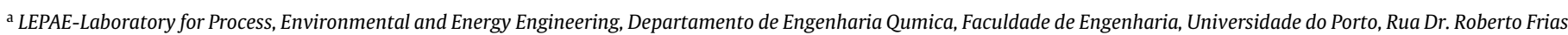 \\ $s / n, 4200-465$ Porto, Portugal \\ ${ }^{\mathrm{b}}$ IBB-Institute for Biotechnology and Bioengineering, Centre of Biological Engineering, Universidade do Minho, Campus de Gualtar, 4710-057 Braga, Portugal
}

\section{A R T I C L E I N F O}

\section{Article history:}

Received 25 June 2010

Received in revised form 18 October 2010

Accepted 30 December 2010

Available online 11 January 2011

\section{Keywords:}

Bubble column

Mass transfer

Absorption

Multiphase reactors

Particles

Slurries

\begin{abstract}
A B S T R A C T
The knowledge about the effects of solids on gas-liquid systems and the respective physical mechanisms are not yet totally clarified. In this work, the effect of the solids on the mass transfer characteristics in a bubble column was studied experimentally for the systems air/water/expandable polystyrene (EPS) beads and air/water/glass beads. Volumetric liquid side mass transfer coefficient, $k_{L} a$, was determined under different solid concentrations (up to $30 \mathrm{vol} \%$ ), superficial gas velocities (up to $2.7 \mathrm{~mm} / \mathrm{s}$ ) and mean diameters (1100, 770 and $591 \mu \mathrm{m}$ for EPS and $9.6 \mu \mathrm{m}$ for glass beads).

The presence of EPS solids affects negatively $k_{L} a$ being this effect more pronounced for the smaller particles. Also, a decrease in $k_{L} a$ occurs when the solid loading increases. Experiments done with large polystyrene particles $\left(d_{p} \geq 591 \mu \mathrm{m}\right)$ contaminated with very fine EPS particles $\left(d_{p} \cong 0.1 \mu \mathrm{m}\right)$ indicate that very fine particles play an important role on gas-liquid mass transfer.

Mass transfer experiments in a hollow glass spheres three-phase slurry showed a dual effect of solids loading on $k_{L} a$, contrarily to what happens with the previous particles. These results can be associated with the different surface properties of the particles studied.

An empirical correlation for $k_{L} a$ on the experimental variables was developed.
\end{abstract}

(c) 2011 Elsevier B.V. All rights reserved.

\section{Introduction}

In many operations of chemical industry processes, one or more components of a gas phase are absorbed into a liquid phase. The phase contacting is often required to trigger reactions among components of the two phases. The complexity and diversity of industrial processes implied that different types of gas-liquid contactors were developed and constructed, such as, bubble columns, pipes/tubes, mechanical agitated tanks, packed columns, plate/tray columns, spray towers, jet (loop) reactors, tubular/ventury ejectors and motionless mixers [1]. Bubble columns are contactors in which a gas or a mixture of gases in the form of a dispersed phase of bubbles moves in a continuous liquid phase. In the liquid, a suspended or fluidized, reactive or catalytic solid can also be present. Thus, in fact, two or three-phase bubble columns exist [2]. These columns can be of different types such as single stage, multi stage, multi channel, with motionless mixers, loop reactors, jet reactors, downflow bubble columns, three-phase fluidized-bed reactors and slurry reactors. Their applications are not limited to the chemical industry; they can be found in biochem-

\footnotetext{
* Corresponding author. Tel.: + 351 225081678; fax: +351225081632.

E-mail address: frocha@fe.up.pt (F. Rocha).
}

ical operations, separation of mixtures by rectification, absorption, wastewater treatment [3] and petrochemical industry. Bubble columns are also gaining increasing importance in the field of biotechnology [4].

Parameters such as phase holdup, flow regime, bubble size distribution, coalescence characteristics, gas-liquid interfacial area, interfacial mass transfer coefficients, heat transfer coefficients and dispersion coefficients influence the bubble column reactors design. The mass transfer coefficient is believed to be the most important design variable, followed by the gas holdup and the axial dispersion coefficient [5].

In three phase systems, the presence of solids affects the gas-liquid mixture in different ways: bubble formation and rise [6,7], axial and radial profiles [8,9], mixing and dispersion, mass transfer [10-13], and gas holdup and flow regimes [14,15].

Despite all the research efforts, the knowledge about the effects of solids on gas-liquid systems and the respective physical mechanisms are not yet clarified. In the present work, volumetric mass transfer coefficients, $k_{L} a$, were obtained for different gas flow rates, solids loading and size. These results were used in order to develop an empirical correlation for $k_{L} a$, taking into account the different experimental variables. The influence of fine particles on $k_{L} a$ was also analyzed, in order to clarify some unexpected results. 


\begin{tabular}{|ll|}
\hline Nomenclature \\
$a$ & gas-liquid interfacial area $\left(\mathrm{m}^{-1}\right)$ \\
$a_{i}$ & empirical parameter, $i=1,2,3,4$ \\
$b$ & linear regression slope $\left(y_{e s t}=c+b x\right)$ \\
$b_{1}$ & linear regression optimum slope for the left hand \\
& side \\
$b_{2}$ & linear regression optimum slope for the right hand \\
$C$ & side \\
$c$ & oxygen concentration in the liquid $(\mathrm{kg} / \mathrm{L})$ \\
$C_{0}$ & linear regression parameter $\left(y_{\text {est }}=c+b x\right)$ \\
$C^{*}$ & oxygen concentration in the liquid at $t=0(\mathrm{~kg} / \mathrm{L})$ \\
$d_{p}$ & particle mean diameter $(\mu \mathrm{m})(\mathrm{mm}$ in $\mathrm{Eq} .(5))$ \\
$e_{S}$ & solid volume fraction \\
$F^{*}$ & Test $F$ parameter \\
$k_{L}$ & liquid-side mass transfer coefficient $(\mathrm{m} / \mathrm{s})$ \\
$k_{L} a$ & volumetric liquid side mass transfer coefficient $\left(\mathrm{s}^{-1}\right)$ \\
$n_{p}$ & number of points for linear regression \\
$n_{p_{1}}$ & number of points for linear regression for the left \\
$n_{p_{2}}$ & hand side \\
$T$ & number of points for linear regression for the right \\
$t$ & hand side \\
$u_{G}$ & temperature $\left({ }^{\circ} \mathrm{C}\right)$ \\
$y$ & time $(\mathrm{s})$ \\
$y_{e s t}$ & superficial gas velocity $(\mathrm{m} / \mathrm{s})$ \\
& experimental value \\
& estimated value $\left(y_{e s t}=c+b x\right)$
\end{tabular}

\section{Experimental}

\subsection{Experimental set-up}

The contact device used to perform the mass transfer experiments, at atmospheric pressure, was the bubble column represented in Fig. 1, with the respective dimensions. The device is a perspex cylindrical column covered by a perspex rectangular box to control the temperature $\left(25^{\circ} \mathrm{C}\right)$ through water circulation. At the bottom, it is located a gas chamber where the gas enters and then passes through a sparger where the bubbles are formed.

The sparger consists of 13 needles with an inner diameter of $0.3 \mathrm{~mm}$. A scheme of the gas sparger is shown in Fig. 2(a). The shape and size $(25 \mathrm{~mm})$ of the needles ensure the formation of small and well-defined bubbles. The needles disposal and the movement of the bubbles enable the suspension of low density solids, and the homogeneity in terms of $\mathrm{O}_{2}$ concentration of the liquid phase. A concave perspex cylindrical piece (Fig. 2(b)), formed by small cones involving each needle, was placed at the column bottom to allow solids circulation in this section avoiding their deposition, and, consequently, the blockage of the orifices.

A flow valve was used to control the air flow rate that was measured by a KDG Mobrey 2-A-150 R rotameter followed by a manometer, where the gauge pressure was kept at the constant value of 1 bar. Before entering the bubble column, the air is saturated in a humidifier. Dissolved oxygen concentration was measured by a Mettler Toledo In Pro $6100 \mathrm{O}_{2}$ sensor (polarographic electrode) connected to a Mettler Toledo $\mathrm{O}_{2} 4100$ dissolved oxygen meter. The oxygen consumption by the electrode is negligible. A data acquisition board was coupled to the $\mathrm{O}_{2}$ meter, enabling to read the $\mathrm{O}_{2}$ concentration on-line.

\subsection{Mass transfer experiments}

Oxygen mass transfer runs were performed in two and threephase systems. Compressed air from air gas cylinder and tap water were used as gas and liquid phases, respectively. Expandable polystyrene (EPS) particles of three different mean diameters, $d_{p},\left(\right.$ Styropor $^{\circledR}$ VEP $124(\mathrm{P} 124)-d_{p}=1100 \mu \mathrm{m}$, Styropor $^{\circledR}$ VEP 324 $(\mathrm{P} 324)-d_{p}=770 \mu \mathrm{m}$ and Styropor ${ }^{\circledR}$ EP $\left.424(\mathrm{P} 424)-d_{p}=591 \mu \mathrm{m}\right)$ with a density of $\rho_{p}=1040-1050 \mathrm{~kg} / \mathrm{m}^{3}$, and hollow glass spheres (with $d_{p}=9.6 \mu \mathrm{m}$, and a density of $\rho_{p}=1100 \mathrm{~kg} / \mathrm{m}^{3}$ ) were used as solid phase. The experiments were performed for several superficial gas velocities, $u_{G}$, (up to $2.7 \mathrm{~mm} / \mathrm{s}$ ), and different solid concentrations (up to $30 \mathrm{vol} . \%$ ). The clear liquid height was $h_{0}=0.2 \mathrm{~m}$ for all experiments (no liquid throughput) and the polarographic electrode $\left(\mathrm{O}_{2}\right.$ probe) was located transversely $0.1 \mathrm{~m}$ from the gas sparger and, approximately, $2 \mathrm{~cm}$ from the column wall.

The experimental procedure is initiated by bubbling nitrogen to deoxygenate the liquid phase. When the dissolved oxygen concentration is approximately zero, humidified air is fed into the column and then the dissolved oxygen concentration variation with time, $t$, is obtained.

The mass balance for oxygen in the liquid is written as:

$\frac{d C}{d t}=k_{L} a\left(C^{*}-C\right)$

where $C^{*}$ and $C$ are, respectively, the oxygen solubility and oxygen concentration in the liquid. Assuming the liquid phase homogeneous and $C_{0}$ the oxygen concentration at $t=0$, the integration of the previous equation leads to:

$\ln \left(C^{*}-C\right)=\ln \left(C^{*}-C_{0}\right)-k_{L} a t$.

The volumetric mass transfer coefficient can now be determined by plotting $\ln \left(C^{*}-C\right)$ against time $(t)$. The experimental results are reproducible with an average relative error of $5 \%$. Further, $k_{L} a$ results were not influenced by the dynamics of the oxygen electrode since, for all runs, it was verified that its response time $(\sim 35 \mathrm{~s})$ was much smaller than the mass transfer time of the system (ranging from 100 to $650 \mathrm{~s}$ ).

\section{Results and discussion}

\section{1. $k_{L}$ a evaluation from experimental data}

An example of data treatment is presented below, for air-water-P424 beads and superficial gas velocity $u_{G}=2.2 \mathrm{~mm} / \mathrm{s}$. The dissolved oxygen concentration curve for this particular experiment is plotted in Fig. 3. One can distinguish three zones on the graph. Zone I, at the beginning, where the $\mathrm{O}_{2}$ concentration is nearly constant, followed by an intense mass transfer zone where the $\mathrm{O}_{2}$ concentration rises fast (Zone II). The last zone (Zone III) appears close to the saturation, when the mass transfer rate starts to decline.

Plotting $\ln \left(C^{*}-C\right)$ against time (Fig. 4), one can observe that the applicability of Eq. (2) is valid except in Zone I, related with the electrode response. So, $k_{L} a$ was determined from the slope in the linear zone. The solubility of oxygen in water $\left(C^{*}\right)$ was taken from the literature [16] and the slope was determined using the statistical method Test $F$ [17]. This method consists in determining the optimum number of points $\left(n_{p}\right)$ for a linear regression of the experimental data. Initially, a short data interval is considered and the linear regression parameters ( $c$ and $b$ ) are determined as well as a parameter $F^{*}$. This parameter is defined as:

$F^{*}=\frac{1}{n_{p}-1} \sqrt{\frac{\sum\left(y_{\text {est }}-y\right)^{2}}{\sum y^{2}}}$ 


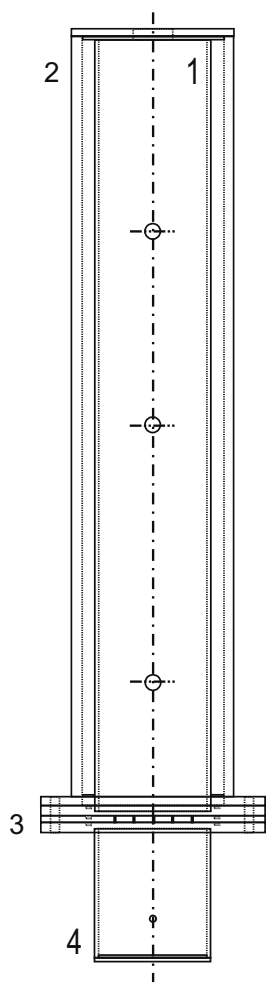

\begin{tabular}{|l|l|r|}
\hline \multicolumn{1}{|c|}{ Component } & Dimensions & $\mathrm{mm}$ \\
\hline \multirow{3}{*}{ - Cylindrical column } & Internal diameter & 84 \\
\cline { 2 - 3 } & Thickness & 3 \\
\cline { 2 - 3 } & High & 600 \\
\hline \multirow{2}{*}{ - Rectangular box } & Width & 110 \\
\cline { 2 - 3 } & Thickness & 8 \\
\cline { 2 - 3 } & High & 600 \\
\hline 3 - Sparger & Thickness & 5 \\
\hline 4 - Gas chamber & Internal diameter & 84 \\
\cline { 2 - 3 } & Thickness & 3 \\
\cline { 2 - 3 } & High & 100 \\
\hline
\end{tabular}

Fig. 1. Bubble column.

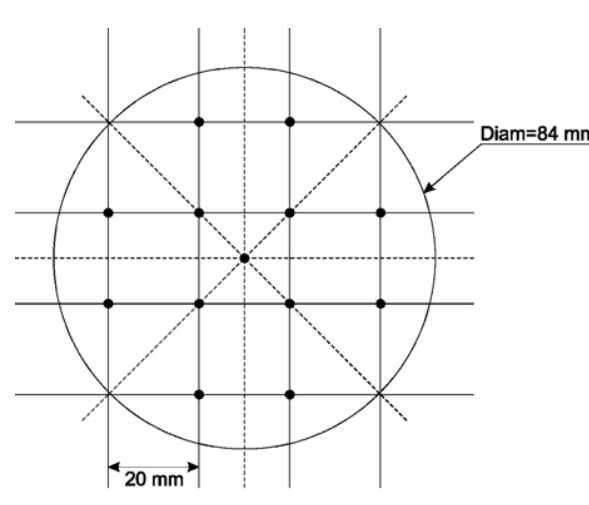

a

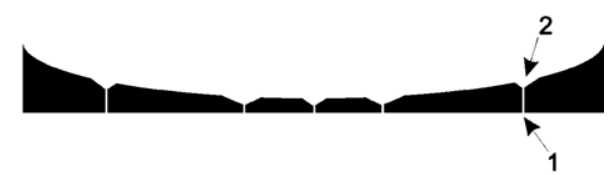

b

Fig. 2. Gas sparger: (a) needles plate for gas sparging; (b) concave perspex cylindrical piece: 1: needle location and 2: cone involving the needle.

where $y$ is the experimental value and $y_{\text {est }}$ is the estimated value $\left(y_{e s t}=c+b x\right)$. At each iteration, an increment in $n_{p}$ is added to the data interval and the parameters $c$ and $b$ are recalculated until the parameter $F^{*}$ reaches its minimum.

Since the linear region is located somewhere in Zone II, first a fixed initial point, located at the middle of this zone, is defined and the Test $F$ is performed to the left and the right hand sides of the initial point (Fig. 4). Two optimum slopes and intervals are obtained and the final value of $k_{L} a$ is the weighted average of the slopes:

$k_{L} a=\left|\frac{n_{p_{1}} b_{1}+n_{p_{2}} b_{2}}{n_{p_{1}}+n_{p_{2}}}\right|$

where $n_{p_{1}}, b_{1}$ and $n_{p_{2}}, b_{2}$ are the optimum number of points and slopes for the left and right hand sides of the initial point, respectively. For this particular case the results are presented in Table 1 , and finally one obtains the volumetric mass transfer coefficient $k_{L} a=4.04 \mathrm{E}-03 \mathrm{~s}^{-1}$.
After that, an empirical correlation for $k_{L} a$ on the experimental variables superficial gas velocity, $u_{G}$, particle mean diameter, $d_{p}$, and solid volume fraction, $e_{S}$, was developed. Only few correlations devoted to the effect of solids characteristics on $k_{L} a$ can be found in literature. Zheng et al. [18] correlated $k_{L} a$ with the axial distance from the distributor, superficial gas and liquid velocities, particle diameter and fractional solid holdup. However, this correlation is not applicable to the present study since we have no liquid input. Among other correlations proposed in the literature (e.g. Midoux et al. [19], Kim and Kim [20], Schumpe et al. [21]), the exponential

Table 1

Optimum parameters.

\begin{tabular}{lll}
\hline & Left & Right \\
\hline$F_{*}$ & $4.26 \mathrm{E}-05$ & $8.28 \mathrm{E}-05$ \\
$n_{p}$ & 35 & 19 \\
$c$ & 2.47 & 2.43 \\
$b$ & $-4.08 \mathrm{E}-03$ & $-3.97 \mathrm{E}-03$ \\
\hline
\end{tabular}




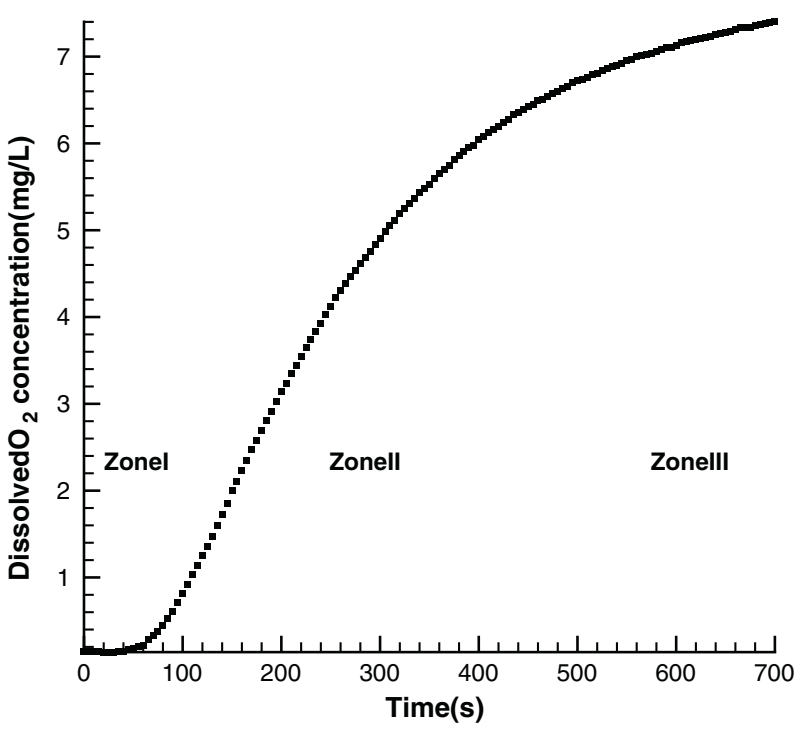

Fig. 3. Dissolved oxygen concentration variation for air-water-P424 beads and $u_{G}=2.2 \mathrm{~mm} / \mathrm{s}$.

dependence was found to be the most suitable. Thus, for each type of solid phase investigated, a correlation of the following type was obtained:

$k_{L} a=a_{1} u_{G}^{a_{2}}\left(1+d_{p}\right)^{a_{3}}\left(1-e_{S}\right)^{a_{4}}$

where $a_{i}$ are empirical parameters, determined by the least squares method for each type of solid phase.

\subsection{Air-water}

Before each three-phase system mass transfer study, air-water two-phase system experiments were performed. The obtained results were compared with correlations presented in literature (Fig. 5). All the correlations displayed can be found in Shah et al. [5], except the one of Sotelo et al. [22]. The majority of the correlations underestimate the experimental values due to differences in the experimental conditions, mainly in superficial gas velocity range and bubble column diameter.

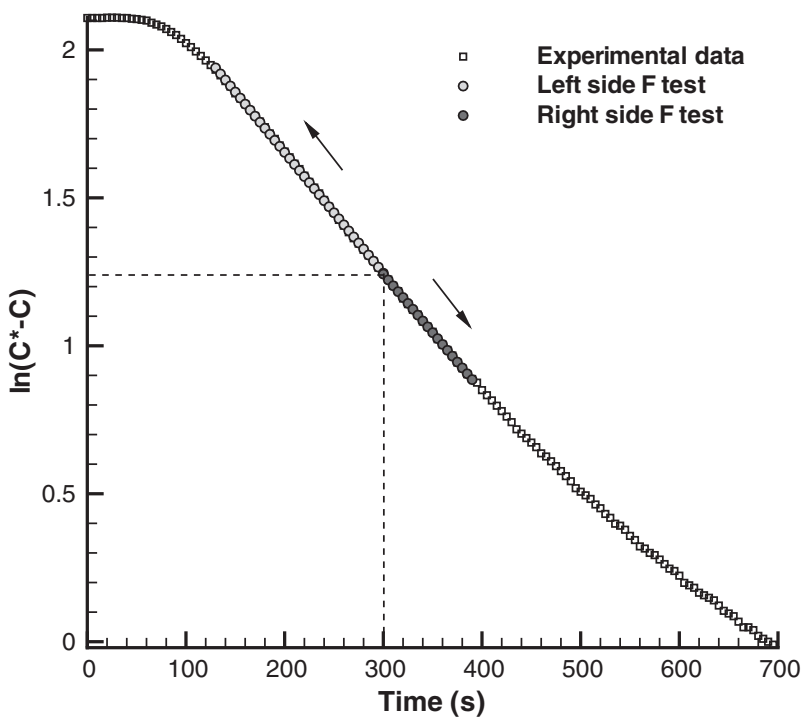

Fig. 4. Test $F$ application for the experimental results for air-water-P424 beads and $u_{G}=2.2 \mathrm{~mm} / \mathrm{s}(C$ in $\mathrm{mg} / \mathrm{L})$.

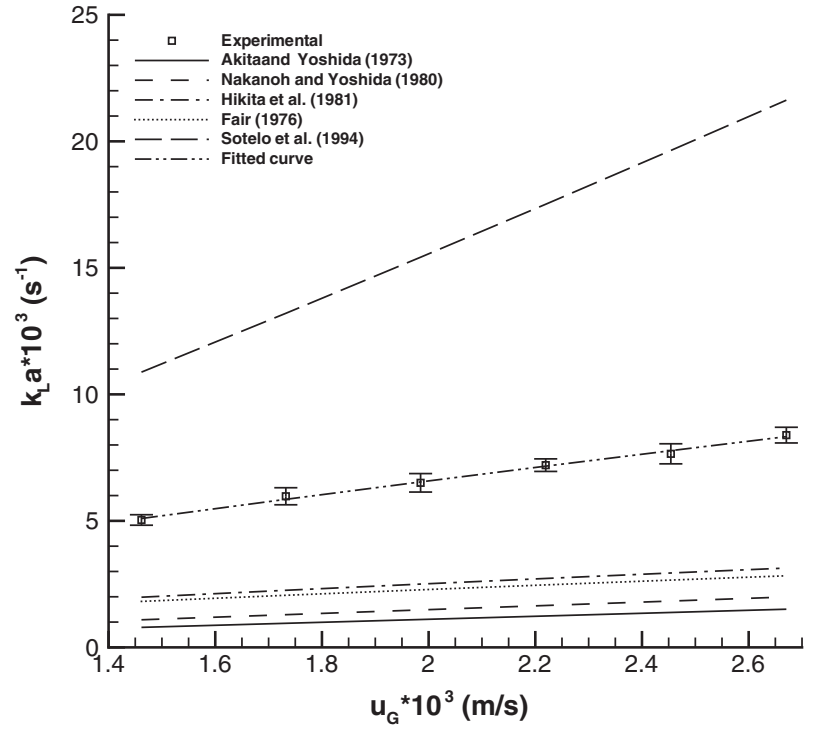

Fig. 5. Dependence of $k_{L} a$ on superficial gas velocity for air-water system.

Overestimation is obtained using the semiempirical equation proposed by Sotelo et al. [22] as a consequence of differences in the type of diffuser. A very good agreement is found with a correlation in the form of $k_{l} a=m u_{G}^{n}$ with parameters $m$ and $n$ equal to 1.05 and 0.82 , respectively. The same type of correlation can be found in literature, as for example in Deckwer et al. [23].

\subsection{Air-water-polystyrene beads}

Two sets of experiments were conducted with polystyrene beads as solid phase. The first set, with polystyrene beads used for the first time, was called "New" polystyrene beads. The second, with polystyrene beads being used after "washing" with air and water in the first experiments, was called "Washed" polystyrene beads. The main difference between these two sets was that, in the first set (with "New" polystyrene beads), fine polystyrene particles from larger beads were being dispersed in the liquid, during the experiments, thus influencing the mass transfer experiments. Consequently, a new set of experiments had to be performed in order to study the influence of fine polystyrene particles on the mass transfer results. For each set, the three particle mean diameters $\left(\mathrm{P} 124 d_{p}=1100 \mu \mathrm{m}\right.$; P32 $4 d_{p}=770 \mu \mathrm{m}$ and P424 $\left.d_{p}=591 \mu \mathrm{m}\right)$ were investigated, for the solid loading range $0-30$ vol.\%.

\subsection{1. "New" polystyrene beads}

Fig. 6(a)-(c) shows how $k_{L} a$ varies with superficial gas velocity, $u_{G}$ and solid loading, for different sizes of "New" polystyrene beads.

It is observed that the volumetric mass transfer coefficient increases with the superficial gas velocity for all particle sizes. In the case of the larger particles $\left(d_{p}=1100 \mu \mathrm{m}\right)$ the influence of $u_{G}$ on $k_{L} a$ decreases as the solid loading increases, being almost negligible for 25 and 30 vol.\% of solids. These conclusions are in agreement with Mena et al. [13] that report a similar effect when calcium alginate beads $\left(d_{p}=1200 \mu \mathrm{m}\right)$ are used as solid phase. According to the authors, $k_{L} a$ decreases with the solid loading increase. However, based in the present results, its seems that this effect is enhanced by the presence of the fine particles.

For intermediate size polystyrene particles $\left(d_{p}=770 \mu \mathrm{m}\right) k_{L} a$ also decreases with the solid loading increase, but in this case only for solid concentrations up to $20 \mathrm{vol} . \%$, remaining constant for further solid content increase. 

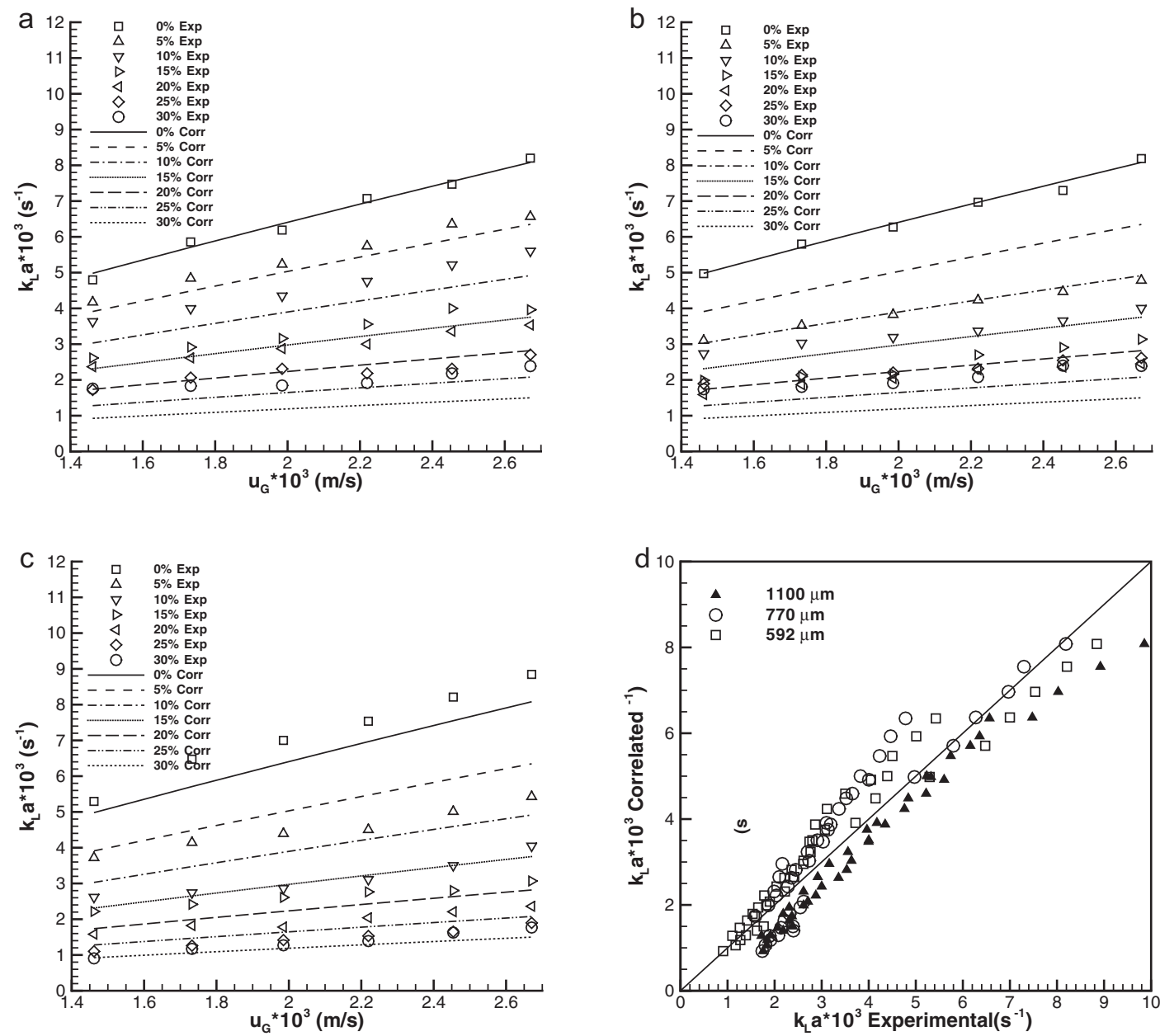

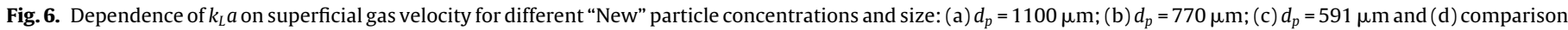
of experimental data and correlation.

The biggest influence of solids loading on $k_{L} a$ was observed during the experiments where the smaller size polystyrene particles $\left(d_{p}=591 \mu \mathrm{m}\right)$ were used (Fig. 6(c)).

With the experimental mass transfer results for "New" polystyrene beads as solid phase and the three particle mean diameters, a general empirical correlation was obtained:

$k_{L} a=0.94 u_{G}^{0.80}\left(1+d_{p}\right)^{0.96}\left(1-e_{S}\right)^{4.72}$

A comparison between experimental and correlated values is plotted in Fig. 6 (d). The correlation seems not to correlate well the experimental $k_{L} a$, which can be attributed to the influence of the fine polystyrene particles. The maximum and mean absolute deviations between the experimental and correlated results are, respectively: $47 \%$ and $17 \%$ for $d_{p}=1100 \mu \mathrm{m} ; 47 \%$ and $21 \%$ for $d_{p}=770 \mu \mathrm{m}$; and $36 \%$ and $15 \%$ for $d_{p}=591 \mu \mathrm{m}$.

\subsection{2. "Washed" polystyrene beads}

Fig. 7(a)-(c) shows how $k_{L} a$ varies with superficial gas velocity, $u_{G}$ and solid loading, for "Washed" polystyrene beads of different sizes.

For all polystyrene particles it was observed that the volumetric mass transfer coefficient increases with the superficial gas velocity. But, in this case, the influence of $u_{G}$ on $k_{L} a$ is not significantly affected by the solid loading. However, this is not the case in the experiments with the "New" polystyrene beads and in other works found in the literature, where it was observed that the effect of $u_{G}$ on $k_{L} a$ decreases as the solid loading increases.
For the smallest "Washed" polystyrene particles, some anomalies for higher solid content and intermediate superficial gas velocities were observed (Fig. 7(c)).

With the experimental mass transfer data for "Washed" polystyrene beads as solid phase and the three particle mean diameters, a general empirical correlation was obtained:

$k_{L} a=1.01 u_{G}^{0.82}\left(1+d_{p}\right)^{0.97}\left(1-e_{S}\right)^{2.73}$.

The parity plot is given in Figs. 7 (d). A much better agreement is found for the case of "Washed" particles, comparing to the results obtained with the "New" ones. The maximum and mean absolute deviations between the experimental and correlated results are, respectively: $24 \%$ and $8 \%$ for $d_{p}=1100 \mu \mathrm{m} ; 26 \%$ and $8 \%$ for $d_{p}=770 \mu \mathrm{m}$; and $29 \%$ and $11 \%$ for $d_{p}=591 \mu \mathrm{m}$. Globally, the mean absolute deviation is $9 \%$, which is near the experimental error (5\%).

Summarizing the main results for air-water-polystyrene systems, it was found that the volumetric mass transfer coefficient increases with the superficial gas velocity. This dependence is generally flattened for increasing solid content. When solid loading increases, the $k_{L} a$ values decrease. With the experimental mass transfer data for "New" and "Washed" polystyrene beads as solid phase, and the three particle mean diameters, two empirical correlations were obtained and a good agreement between correlated and experimental data was verified for the "Washed" polystyrene beads. These results indicate that the mechanisms associated with the large and very fine particles are not the same. 

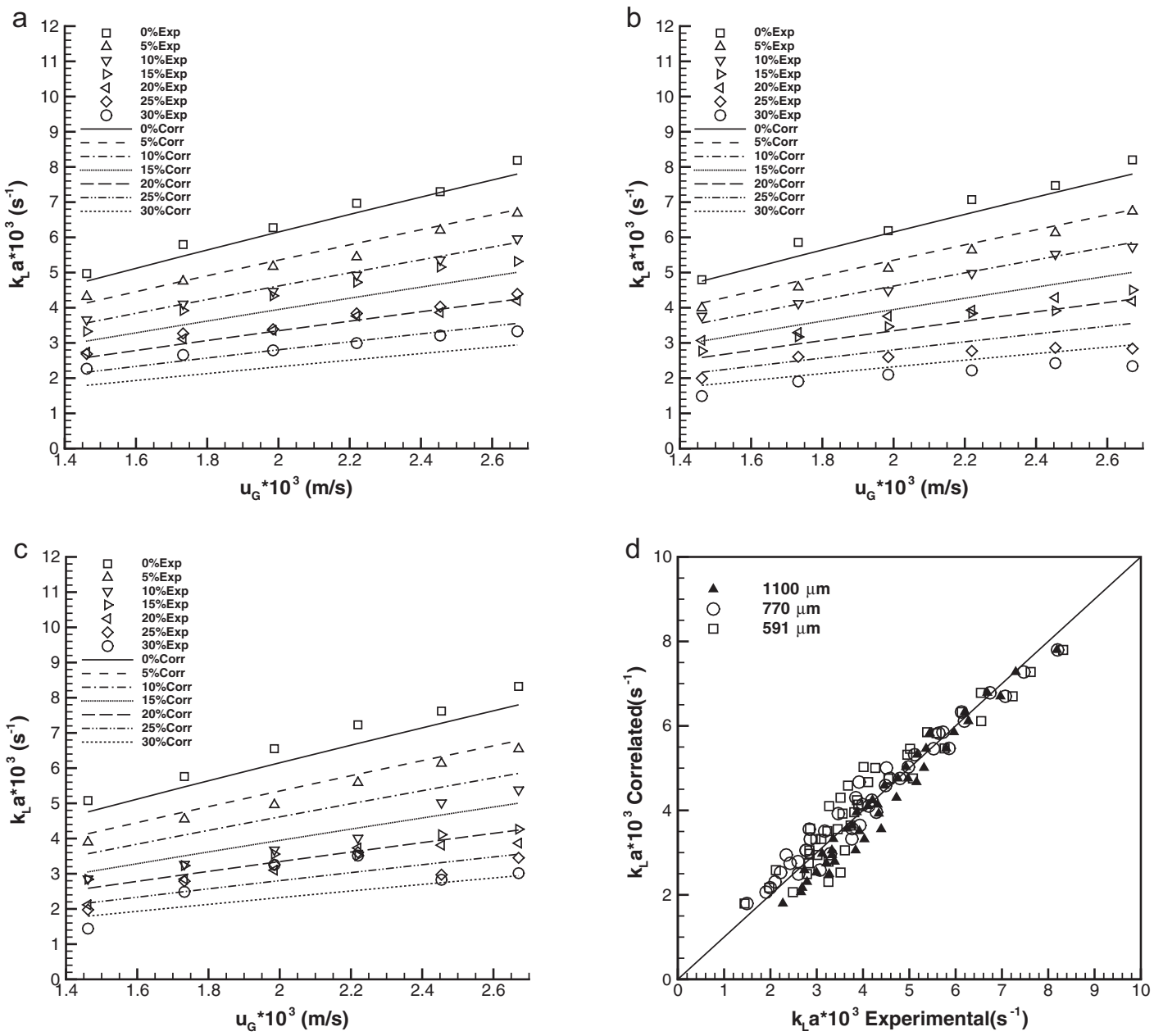

Fig. 7. Dependence of $k_{L} a$ on superficial gas velocity for different "Washed" particle concentrations and size: (a) $d_{p}=1100 \mu \mathrm{m}$; (b) $d_{p}=770 \mu \mathrm{m}$; (c) $d_{p}=591 \mu \mathrm{m}$ and (d) comparison of experimental data and correlation.

Shah at al. [5] referred that the effect of solid concentration on $k_{L} a$ strongly depends on the gas and liquid velocities. In the present study, the liquid velocity is zero, but one noticed that the negative solid influence on $k_{L} a$ is stronger for higher gas velocities. In spite of the low gas holdup $(\approx 1 \%)$, for higher gas velocities, more bubbles are in the bubble column at a certain instant. Thus, and taking into account the high solid fraction (up to 30 vol.\%), the probability of bubble-bubble interaction increases (with both $u_{G}$ and $e_{S}$ ), which may increase the bubble coalescence rate. The gas-liquid interfacial area decreases which leads to a reduction on the volumetric mass transfer coefficient. Freitas and Teixeira [24] also observed a $k_{L} a$ reduction with the increase in solids (calcium alginate beads) loading, especially for high superficial gas velocities. This effect was also attributed to an increase in bubble coalescence. According to Nguyen-Tien at al. [25] for small particles $\left(d_{p} \leq 1 \mathrm{~mm}\right), k_{L} a$ decreases with solid volume fraction. This behaviour was explained by the increase in the suspension viscosity that causes bubble coalescence. The larger beads used in the present work had $d_{p}=1100 \mu \mathrm{m}$, so we can consider that we are roughly within the particle size range defined above, which confirms the agreement between the present results with those presented by Nguyen-Tien at al. [25].

The effect of particle mean diameter on the mass transfer coefficient is shown in Fig. 7, for each solid loading, and for the "Washed" polystyrene beads. The influence of particle mean diameter is negligible for the case of $5 \mathrm{vol}$ \% of solids. For the rest of solid loadings, the general trend indicates that the decrease of particle mean diameter results in a reduction of the mass transfer coefficient. Kim and Kim [20] mentioned a similar influence of particle size on $k_{L} a$, but an opposite effect was reported by Dhanuka and Stepanek [26] and Zheng at al. [18]. These differences can be attributed to the differences in experimental conditions and particulary in solid type (namely: hydrophobicity, geometry and density) and particle size range.

\subsection{Effect of fine polystyrene particles on $k_{L} a$}

As already mentioned, during the mass transfer experiments, the "New" polystyrene spheres released very fine particles $\left(d_{p} \cong 0.1 \mu \mathrm{m}\right)$ which were dispersed in the liquid phase. Fig. 8 shows a comparison between $k_{L} a$ values for the experiments with "New" and "Washed" polystyrene beads.

Mass transfer coefficients for "Washed" particles experiments are generally substantially higher than those found for correspondent experiments with "New" particles, which means that the fine particles influence negatively the mass transfer process. These particles, whose size is smaller than the liquid film around the bubble $(\delta \leq 20 \mu \mathrm{m})$, are hydrophobic. Therefore, they tend to stick on the bubble, partially covering its surface and blocking the mass transfer path. As a consequence, the liquid side mass transfer coefficient $\left(k_{L}\right)$ and the specific interfacial area $(a)$ are reduced and the volumetric mass transfer coefficient $\left(k_{L} a\right)$ decreased. 


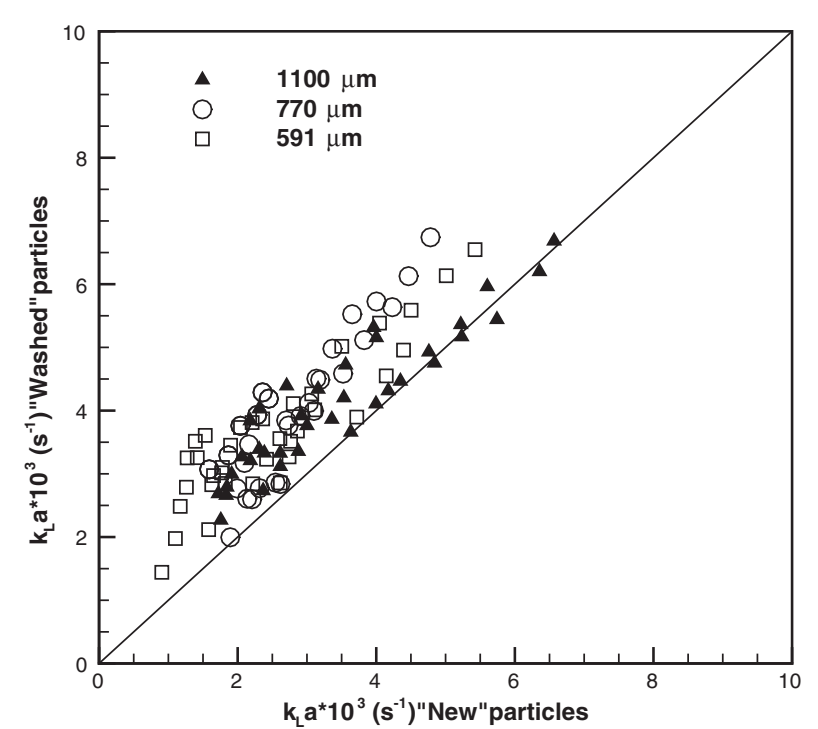

Fig. 8. Effect of polystyrene fine particles on $k_{L} a$ for air-water-polystyrene systems.

\subsection{Air-water-hollow glass spheres}

In order to study in more detail the influence of fine particles on $k_{L} a$, mass transfer experiments were conducted in a slurry composed by $9.6 \mu \mathrm{m}$ hollow glass spheres as solid phase, for concentrations up to $15 \mathrm{vol} . \%$. As in the previous experiments, the mass transfer coefficient increases with the superficial gas velocity. The same type correlation of Eq. 5 was tested but without success. In this case the influence of solid phase on mass transfer is quite different of the previous one (Section 3.3.2). Here the particle size is much smaller and surface properties are different. So, the physical mechanisms governing the solid effect on $k_{L} a$ could be also different.

A dual effect of solids loading on $k_{L} a$ is shown in Fig. 9. For low solids loading ( $\leq 3 \mathrm{vol} . \%$ ) the mass transfer coefficient increases with the solid content and then decreases with further solid additions (Fig. 9). Two possible reasons can be pointed out for the enhancement of $k_{L} a$ with the solid loading increase verified for low solid contents: the first is the fact that small solid concentrations do not change significantly the liquid viscosity, but improve

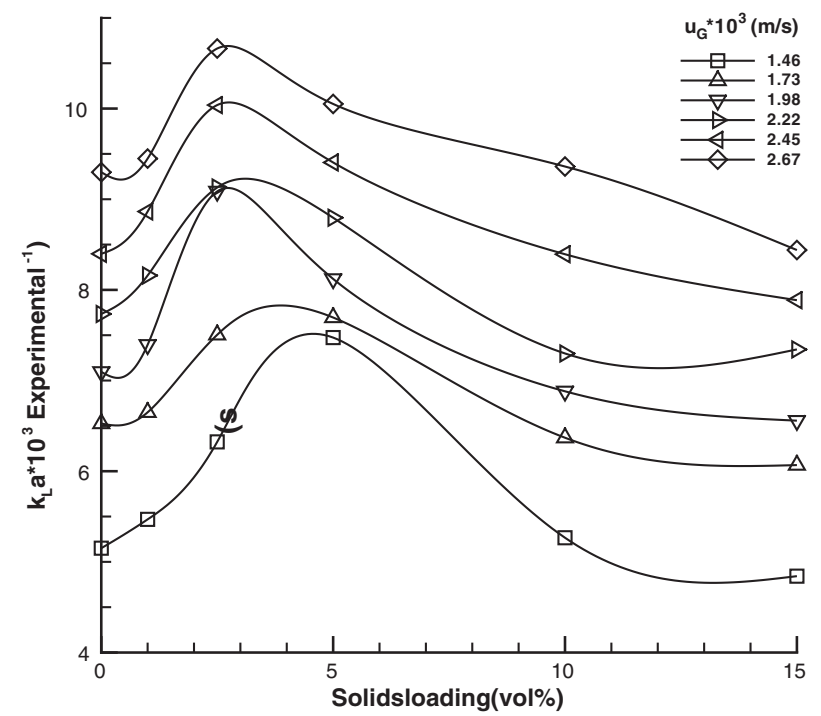

Fig. 9. Experimental volumetric mass transfer coefficient as a function of hollow glass spheres loading. the surface renewal and turbulence in the liquid film, increasing $k_{L}$ and thus $k_{L} a$; the second is associated with the presence of fine particles in the liquid film at gas-liquid interface which may hinder the coalescence behaviour of bubbles, consequently increasing the gas-liquid interfacial area. On the other hand, higher solid concentrations increase the slurry viscosity and hence decreasing the surface renewal and mobility which results in reduced $k_{L}$. In addition, for higher solid contents, the gas-liquid interface will be covered by fine particles, hindering the oxygen diffusion and reducing $k_{L}$. Also, a reduction of gas holdup with the solid content increase is expected, which consequently decreases the g-l interfacial area, $a$. Indeed, as mentioned before, the effective mixture viscosity increases as the solid load increases which will induce the formation of bigger bubbles at the gas distributor, promoting the bubble coalescence and suppressing the bubble breakup [5]. Therefore, both decreases of $k_{L}$ and $a$ obviously result in a reduction of the mass transfer coefficient $k_{L} a$.

Similar dual effect of solids on $k_{L} a$ was reported by Ozkan at al. [27] and Albal et al. [28]. However, reduction of $k_{L} a$ with an increase of solid loading for a certain solid content range was referred by Sada et al. [29] and Zahradnik et al. [30], while Chandrasekaran and Sharma [31], Quicker et al. [10] and Sada et al. [11] presented increases in the $k_{L} a$ with the solid loading. Once again, these discrepancies may arise from differences in experimental conditions, such as physicochemical properties of the liquid and also, loading, size, density and wettability of solids. Further investigations are needed on possible mechanisms based on changes in the gas phase (gas holdup and bubble coalescence) and on the environment around bubble (interaction of liquid film and fine particles, surface tension at the bubble, liquid film and particle interfaces, wetting differences, adsorption) [27].

\section{Conclusions}

The gas-liquid mass transfer process was investigated in a three-phase bubble column. The main purpose was to analyze the effect of certain solid properties on the gas-liquid mass transfer. The solid characteristics under study were the mean diameter, loading and type.

Concerning the size and loading effects three behaviours were observed, according to particle mean diameter:

- $590<d_{p}<1100 \mu \mathrm{m}$ :

It was found that $k_{L} a$ increases with the superficial gas velocity $u_{G}$, and this dependence generally flattens for increasing solid content. The effect of particle mean diameter on $k_{L} a$ is not constant but, in general, $k_{L} a$ decreases as the particle mean diameter decreases. When the solid loading increases, the $k_{L} a$ values decrease.

The empirical correlation of the type: $k_{L} a=$ $a_{1} u_{G}^{a_{2}}\left(1+d_{p}\right)^{a_{3}}\left(1-e_{S}\right)^{a_{4}}$ was developed and a good agreement between correlated and experimental data was verified. The mean absolute deviation obtained was, approximately, 9\%, a value near the experimental result.

- Fine particles $\left(d_{p}=9.6 \mu \mathrm{m}\right)$ :

Mass transfer experiments in a three-phase slurry of $9.6 \mu \mathrm{m}$ hollow glass spheres showed a dual effect of solids loading on $k_{L} a$. For low solids loading ( $\leq 3 \mathrm{vol} . \%$ ) $k_{L} a$ increases with the solid content and then decreases with further solid additions. These results show that $k_{L} a$ can also be enhanced by the presence of the solid phase.

- Very fine particles $\left(d_{p}=0.1 \mu \mathrm{m}\right)$ :

The effect of very fine particle on mass transfer was studied as a contamination of the systems air/water/expandable polystyrene (EPS) beads $\left(d_{p}=1100 \mu \mathrm{m} ; d_{p}=770 \mu \mathrm{m}\right.$ and $\left.d_{p}=591 \mu \mathrm{m}\right)$. The 
results showed the negative effect of these fine particles on $k_{L} a$. It seems that these particles act as an impurity on bubble surface, covering it and blocking, by this way, the mass transfer.

In relation to the effect of surface properties of particles in mass transfer, it was possible to observe that particles, with different surface properties (EPS, hydrophobic, and glass spheres, hydrophilic), can have opposite effects on $k_{L} a$. It seems that hydrophobic particles have a strong negative influence on mass transfer, and hydrophilic particles, in a certain concentration range, can increase $k_{L} a$.

In short, the present results show that the physical mechanisms governing the solid effect on $k_{L} a$ are not limited to the physical and chemical characteristics of the liquid and gas, but also to the solid characteristics, as its size and surface properties.

\section{Acknowledgements}

This work was supported by Fundação para a Ciência e Tecnologia under program contract number SFRH/BD/3427/2000, POCTI/EQU/45194/2002 and SFRH/BPD/45637/2008.

\section{References}

[1] S.-Y. Lee, Y.P. Tsui, Succeed at gas/liquid contracting, Chem. Eng. Prog. 95 (1999) 23-49.

[2] W.-D. Deckwer, A. Schumpe, Bubble columns - the state of the art and current trends, Int. Chem. Eng. 27 (1987) 405-422.

[3] W. Hong, H. Brauer, Gas-liquid mass transfer in bubble-column reactors, Int. Chem. Eng. 29 (1989) 388-434

[4] E. Alvarez, B. Sanjurjo, A. Cancela, J.M. Navaza, Mass transfer and influence of physical properties of solutions in a bubble column, Chem. Eng. Res. Des. 78 (2000) 889-893.

[5] Y.T. Shah, B.G. Kelkar, S.P. Godbole, W.D. Deckwer, Design parameters estimations for bubble column reactors, AIChE J. 28 (1982) 353-379.

[6] G. Yang, B. Du, L. Fan, Bubble formation and dynamics in gas-liquid-solid fluidization - a review, Chem. Eng. Sci. 62 (1-2) (2007) 2-27.

[7] R. Lau, R. Mo, W.S.B. Sim, Bubble characteristics in shallow bubble column reactors, Chem. Eng. Res. Des. 88 (2) (2010) 197-203.

[8] W. Warsito, L.S. Fan, Measurement of real-time flow structures in gas-liquid and gas-liquid-solid flow systems using electrical capacitance tomography (ECT), Chem. Eng. Sci. 56 (2001) 6455-6462.

[9] P. Mena, F. Rocha, J. Teixeira, P. Sechet, A. Cartellier, Measurement of gas phase characteristics using amonofibre optical probe in a three-phase flow, Chem. Eng. Sci. 63 (2008) 4100-4115.

[10] G. Quicker, A. Schumpe, W.D. Deckwer, Gas-liquid interfacial areas in a bubble column with suspended solids, Chem. Eng. Sci. 39 (1984) 179-183.
[11] E. Sada, H. Kumazawa, C. Lee, N. Fujiwara, Gas-liquid mass transfer characteristics in a bubble column with suspended sparingly soluble fine particles, Ind. Eng. Chem. Process Des. Dev. 24 (1985) 255-261.

[12] C. Joly-Vuillemin, C. de Bellefon, H. Delmas, Solid effects on gas-liquid mass transfer in three-phase slurry catalytic hydrogenation of adiponitrile over raney nickel, Chem. Eng. Sci. 51 (1996) 2149-2158.

[13] P.C. Mena, M.C. Pons, J.A. Teixeira, F.A. Rocha, Using image analysis in the study of multiphase gas absorption, Chem. Eng. Sci. 60 (2005) 51445150.

[14] J.P. Zhang, J.R. Grace, N. Epstein, K.S. Lim, Flow regime identification in gas-liquid flow and three-phase fluidized beds, Chem. Eng. Sci. 52 (1997) 3979-3992.

[15] P.C. Mena, F.A. Rocha, J.A. Teixeira, J. Drahos, M.C. Ruzicka, Effect of solids on homogeneous-heterogeneous flow regime transition in three-phase bubble columns, Chem. Eng. Sci. 60 (2005) 6013-6026.

[16] C. Velz, Applied Stream Sanitation, Wiley Interscience, New York, 1970.

[17] P.G. Hoel, Elementary Statistics, 4th Edition, John Wiley \& Sons Inc, Australia, 1976.

[18] C. Zheng, Z. Chen, Y. Feng, H. Hofmann, Mass transfer in different flow regimes of three-phase fluidized beds, Chem. Eng. Sci. 50 (1995) 1571-1578.

[19] N. Midoux, B.I. Morsi, M. Purwasasmita, A. Laurent, J.C. Charpentier, Interfacial area and liquid side mass transfer coefficient in trickle bed reactors operating with organic liquids, Chem. Eng. Sci. 39 (1984) 781-794.

[20] J.O. Kim, S.D. Kim, Gas-liquid mass transfer in a three-phase fluidized bed with floating bubble breakers, Can. J. Chem. Eng. 68 (1990) 368-375.

[21] A. Schumpe, W.-D. Deckwer, K.D.P. Nigam, Gas-liquid mass transfer in threephase fluidized beds with viscous pseudoplastic liquids, Can. J. Chem. Eng. 67 (1989) 873-877.

[22] J.L. Sotelo, F.J. Benitez, J. Beltran-Heredia, C. Rodriguez, Gas holdup and mass transfer coefficients in bubble columns. 1. Porous glass-plate diffusers, Int. Chem. Eng. 34 (1) (1994) 82-90.

[23] W.D. Deckwer, R. Burckhart, G. Zoll, Mixing and mass transfer in tall bubble columns, Chem. Eng. Sci. 29 (1974) 2177-2188.

[24] C. Freitas, J.A. Teixeira, Oxygen mass transfer in a high solids loading threephase internal-loop airlift reactor, Chem. Eng. J. 84 (2001) 57-61.

[25] K. Nguyen-Tien, A.N. Patwari, A. Schumpe, W.D. Deckwer, Gas-liquid mass transfer in fluidized particle beds, AIChE J. 31 (1985) 194-201.

[26] V.R. Dhanuka, J.B. Stepanek, Simultaneous measurement of interfacial area and mass transfer coefficient in three phase fluidized beds, AIChE J. 26 (1980) 1029-1038.

[27] O. Ozkan, A. Calimli, R. Berber, H. Oguz, Effect of inert solid particles at low concentrations on gas-liquid mass transfer in mechanically agitated reactors, Chem. Eng. Sci. 55 (2000) 2737-2740.

[28] R.S. Albal, Y.T. Shah, A. Schumpe, Mass transfer in multiphase agitated contactors, Chem. Eng. J. 27 (1983) 61-80.

[29] E. Sada, H. Kumazawa, C.H. Lee, Chemical absorption in a bubble column loading concentrated slurry, Chem. Eng. Sci. 38 (1983) 2047-2051.

[30] J. Zahradnik, L. Drapal, F. Kastánek, J. Reznickova, Hydrodynamic and mass transfer characteristics of sectionalized aerated slurry reactors, Chem. Eng. and Process. 31 (1992) 263-272.

[31] K. Chandrasekaran, M.M. Sharma, Absorption of oxygen in aqueous solutions of sodium sulfide in the presence of activated carbon as catalyst, Chem. Eng. Sci. 32 (1977) 669-671. 